\title{
NUDELMAN INTERPOLATION, PARAMETRIZATIONS OF LOSSLESS FUNCTIONS AND BALANCED REALIZATIONS.
}

\author{
Jean-Paul Marmorat* Martine Olivi** \\ * CMA, BP 93, 06902 Sophia-Antipolis Cedex, FRANCE, \\ marmorat@sophia.inria.fr, phone: 334923879 56, fax: \\ 33492387998 \\ ** INRIA, BP 93, 06902 Sophia-Antipolis Cedex, \\ FRANCE, olivi@sophia.inria.fr, phone:334923878 77, \\ fax: 33492387858
}

\begin{abstract}
We investigate the parametrization issue for discrete-time stable allpass multivariable systems by means of a Schur algorithm involving a Nudelman interpolation condition. A recursive construction of balanced realizations is associated with it, that possesses a very good numerical behavior. Several atlases of charts or families of local parametrizations are presented and for each atlas a chart selection strategy is proposed. The last one can be viewed as a nice mutual encoding property of lossless functions and turns out to be very efficient. These parametrizations allow for solving optimization problems within the fields of system identification and optimal control.
\end{abstract}

Keywords: Parametrization, Inner matrices, Interpolation algorithms, Scattering parameters, Differential geometric methods, Discrete-time systems, Multivariables systems.

\section{INTRODUCTION}

Lossless or stable allpass transfer functions play an important role in system theory mainly due to the Douglas-Shapiro-Shields factorization: any proper transfer function can be written as the product of a lossless function, which includes the dynamics of the system, and an unstable factor. In many problems in which a criterion must be optimized over a set of functions, the unstable factor of the optimum can be computed from the lossless one. This can be done in rational $L^{2}$ approximation (Fulcheri and Olivi [1998]), system identification (Bruls et al. [1999]), multi-objective control (Scherer [2000]). These problems can thus be handled by optimization methods over the class of lossless functions of prescribed degree, or possibly a specified subclass. It is with such applications in mind that we will address the parametrization issue.

An interesting and unusual approach of this optimization problems is to use the manifold structure of the class of lossless functions of fixed McMillan degree (Alpay et al. [1994]) and parameters coming from an atlas of charts. An atlas of charts attached with a manifold is a collection of local coordinate maps (the charts), whose domains cover the manifold and such that the changes of coordinates are smooth. Using such parameters allows to exactly describe the set on which an optimum is searched. This ensures that the optimum will be stable and of the prescribed order. In practice, a search algorithm can be run through 
the manifold as a whole, using a local coordinate map to describe it locally and changing from one coordinate map to another when necessary.

In the literature, atlases of charts have been derived both from the state-space approach using nice selections and from the functional approach using interpolation theory and Schur type algorithms. A connection between these two approaches was found in the scalar (or SISO) case (Hanzon and Peeters [2000]) and generalized to the matrix case (Hanzon et al. [2004]). In this paper, an atlas is described in which balanced realizations can be computed from the Schur parameters. The computation involves a product of unitary matrices and thus presents a nice numerical behavior. Moreover, for some particular choices of the interpolation points and directions, the balanced realizations possess a triangular structure which relates to nice selections (Peeters et al. [2004]).

The natural framework for these studies is that of complex functions. However, systems are often real-valued and their transfer functions $T$ are real, that is, they satisfy the relation $\overline{T(z)}=T(\bar{z})$. Even if the complex case includes the real case by restriction, a specific treatment is actually relevant and was the initial motivation for this work which notably improves Marmorat et al. [2003]. In rational $L^{2}$ approximation for example, a real function may have a complex best approximant. This is the case for the function $f(z)=1 / z^{3}$ $1 / z$ which admits three minima: a real one and two complex ones, which achieve the best relative error.

In this paper, atlases are constructed in which lossless functions are represented by balanced realizations built recursively from interpolation data as in Hanzon et al. [2004]. But instead of the Nevanlinna-Pick interpolation problem used there we consider here the more general Nudelman interpolation problem. This very general framework allows to construct several atlases, including that of Hanzon et al. [2004], and to describe the subclass of real functions. For each particular atlas presented in this work, we propose a simple method to find an "adapted chart" for a given lossless function. This last point, together with their nice numerical behavior, make these parametrizations an interesting tool for solving the optimization problems mentioned before.

\section{PRELIMINARIES.}

This paper is concerned with finite dimensional, stable, discrete-time systems and their transfer functions which happen to be rational functions analytic outside the closed unit disk. Interpolation theory usually deals with functions that are analytic in the open unit disk. To relate these two situations, we use the transformation $F \rightarrow F^{\sharp}$ defined by

$$
F^{\sharp}(z)=F^{*}(1 / z), \quad F^{*}(z)=F(\bar{z})^{*} .
$$

Let

$$
J=\left[\begin{array}{cc}
I_{p} & 0 \\
0 & -I_{p}
\end{array}\right]
$$

A $2 p \times 2 p$ rational matrix function $\Theta(z)$ is called $J$-lossless (or conjugate $J$-inner) if, at every point of analyticity $z$ of $\Theta(z)$ it satisfies

$$
\begin{array}{ll}
\Theta(z) J \Theta(z)^{*} \leq J, & |z|>1 \\
\Theta(z) J \Theta(z)^{*}=J, & |z|=1
\end{array}
$$

The simplest $J$-lossless functions are the constant $J$ unitary matrices $H$ satisfying $H^{*} J H=J$.

A $p \times p$ rational matrix function $G(z)$ is called lossless or conjugate inner (resp. inner), if and only if

$$
G(z) G(z)^{*} \leq I_{p}, \quad|z|>1(\text { resp. }|z|<1),
$$

with equality on the circle. The transfer function of a lossless system is a lossless function. A lossless function can have no pole on the unit circle and the identity $G^{\sharp}(z) G(z)=I_{p}$ for $|z|=1$, extends by analytic continuation to all points where both $G(z)$ and $G^{\sharp}(z)$ are analytic. Therefore, the function $G(z)^{-1}$ agrees with $G^{\sharp}(z)$ and is inner.

We denote by $\mathcal{L}_{n}^{p}$ the set of $p \times p$ lossless functions of McMillan degree $n$, by $\mathcal{R} \mathcal{L}_{n}^{p}$ the subset of real functions and by $\mathbb{U}(p)$ the set of $p \times p$ constant unitary matrices. The McMillan degree will be denoted by deg.

An important property of a lossless function is that if

$$
G(z)=C\left(z I_{n}-A\right)^{-1} B+D
$$

is a balanced realization (it always exists, see Genin et al. [1983]), then the associated realization matrix

$$
R=\left[\begin{array}{ll}
D & C \\
B & A
\end{array}\right]
$$

is unitary. Lossless functions can thus be represented by unitary realization matrices. Conversely, if the realization matrix associated with a realization of order $n$ of some $p \times p$ rational function $G(z)$ is unitary, then $G(z)$ is lossless of McMillan degree less or equal to $n$. For these questions, we refer the reader to Hanzon et al. [2004] and the bibliography therein. 
Along with a $2 p \times 2 p$ rational function $\Theta(z)$ blockpartitioned as follows

$$
\Theta(z)=\left[\begin{array}{cc}
\Theta_{11}(z) & \Theta_{12}(z) \\
\Theta_{21}(z) & \Theta_{22}(z)
\end{array}\right]
$$

with each block of size $p \times p$, we associate the linear fractional transformation $T_{\Theta}$ which acts on $p \times p$ rational functions $F(z)$ as follows:

$$
T_{\Theta}(F)=\left[\Theta_{11} F+\Theta_{12}\right]\left[\Theta_{21} F+\Theta_{22}\right]^{-1} .
$$

For a composition of linear fractional transformations, it holds that $T_{\Theta} \circ T_{\Psi}=T_{\Theta \Psi}$. Linear fractional transformations occur extensively in representation formulas for the solution of various interpolation problems (Ball et al. [1990]). To adapt the results available in the literature for functions analytic in the disk to the case of functions analytic outside, we use the relation

$$
Q=T_{\Theta}(R) \Leftrightarrow Q^{\sharp}=T_{J_{1} \Theta J_{1}}\left(R^{\sharp}\right), J_{1}=\left[\begin{array}{cc}
0 & I_{p} \\
I_{p} & 0
\end{array}\right] .
$$

In particular, we have the following result, stated for inner functions for example in [Fulcheri and Olivi, 1998, Lemma 3]:

Theorem 1. If $\Theta(z)$ is a $J$-lossless matrix function, then the map $T_{\Theta}$ sends every lossless function to a lossless function.

\section{NUDELMAN INTERPOLATION FOR LOSSLESS FUNCTIONS}

The Nudelman interpolation problem is to find a $p \times p$ rational lossless function $G(z)$ which satisfies an interpolation condition of the form

$$
\frac{1}{2 i \pi} \int_{\mathbf{T}} G^{\sharp}(z) U\left(z I_{\delta}-W\right)^{-1} d z=V,
$$

where $(U, W)$ is an observable pair and $W$ is stable ( $U$ is $p \times \delta$ and $W$ is $\delta \times \delta$ ). Note that if $W$ is a diagonal matrix, this problem reduces to a Nevanlinna-Pick problem.

It is well-known that there exists a rational lossless function $G(z)$ satisfying the interpolation condition (8) if and only if the solution $P$ of the symmetric Stein equation

$$
P-W^{*} P W=U^{*} U-V^{*} V
$$

is positive definite [Ball et al., 1990, Th.18.5.2]. A triple $(W, U, V)$ such that the solution $P$ of (9) is positive definite, will be called an admissible Nudelman data set. A $2 p \times 2 p J$-lossless function can then be built from $(W, U, V)$ :

$\Theta_{W, U, V}(z)=$

$\left[I_{2 p}-(z-1) C\left(z I_{\delta}-W\right)^{-1} P^{-1}\left(I_{\delta}-W\right)^{-*} C^{*} J\right]$ where $C=\left[\begin{array}{l}U \\ V\end{array}\right]$.

Theorem 2. Let $(W, U, V)$ be an admissible Nudelman data set, and let $\Theta=\Theta_{W, U, V} H$, where $\Theta_{W, U, V}$ is given by (10) and $H$ is an arbitrary constant $J$-unitary matrix. For every lossless function $F(z)$, the lossless function

$$
G=T_{\Theta}(F)
$$

satisfies (8) and $\operatorname{deg} G=\operatorname{deg} F+\delta$. Conversely, the set of all lossless solutions $G(z)$ of $(8)$ is given by (11) where $F(z)$ is an arbitrary lossless function.

Proof. This result is a particular case of [Ball et al., 1990, Th.18.5.2], which describes all the Schur functions which are solutions of a Nudelman interpolation problem.

Let $\Lambda$ and $\Pi$ be $p \times p$ unitary matrices. Then the following important relations are satisfied

$$
\begin{gathered}
{\left[\begin{array}{cc}
\Lambda & 0 \\
0 & \Pi
\end{array}\right] \Theta_{W, U, V}\left[\begin{array}{cc}
\Lambda^{*} & 0 \\
0 & \Pi^{*}
\end{array}\right]=\Theta_{W, \Lambda U, \Pi V}} \\
T_{\Theta_{W, \Lambda U, \Pi V}}\left(\Lambda F(z) \Pi^{*}\right)=\Lambda T_{\Theta_{W, U, V}}(F(z)) \Pi^{*}
\end{gathered}
$$

\section{BALANCED REALIZATIONS}

The aim of this section is to choose the arbitrary $J$-unitary factor $H$ in Theorem 2, so that the linear fractional transformation $\widetilde{G}=T_{\Theta_{W, U, V} H}(G)$ yields a simple and powerful construction for balanced realizations as in (Hanzon et al. [2004]).

Let $\mathcal{U}$ and $\mathcal{V}$ be $(p+\delta) \times(p+\delta)$ unitary matrices partitioned as follows:

$$
\mathcal{U}=\left[\begin{array}{cc}
M_{u} & \alpha_{u} \\
\beta_{u}^{*} & K_{u}
\end{array}\right], \quad \mathcal{V}=\left[\begin{array}{cc}
M_{v} & \alpha_{v} \\
\beta_{v}^{*} & K_{v}
\end{array}\right],
$$

where $K_{u}$ and $K_{v}$ are $\delta \times \delta, \alpha_{u}, \alpha_{v}, \beta_{u}$ and $\beta_{v}$ are $p \times \delta$ and $M_{u}$ and $M_{v}$ are $p \times p$, and put

$$
M=\left[\begin{array}{cc}
M_{u} & 0 \\
0 & M_{v}
\end{array}\right], \quad \alpha=\left[\begin{array}{l}
\alpha_{u} \\
\alpha_{v}
\end{array}\right], \quad \beta=\left[\begin{array}{l}
\beta_{u} \\
\beta_{v}
\end{array}\right] .
$$

Proposition 1. Let $\mathcal{U}$ and $\mathcal{V}$ be unitary matrices block-partitioned as in (14). Assume that $K_{v} z-$ $K_{u}$ is invertible. Given a $p \times p$ proper rational transfer function $G(z)$ and a minimal realization $G(z)=D+C\left(z I_{k}-A\right)^{-1} B$, the formula

$$
\left[\begin{array}{cc}
\widetilde{D} & \widetilde{C} \\
\widetilde{B} & \widetilde{A}
\end{array}\right]=\left[\begin{array}{cc}
\mathcal{U} & 0 \\
0 & I_{k}
\end{array}\right]\left[\begin{array}{ccc}
D & 0 & C \\
0 & I_{\delta} & 0 \\
B & 0 & A
\end{array}\right]\left[\begin{array}{cc}
\mathcal{V}^{*} & 0 \\
0 & I_{k}
\end{array}\right],
$$

in which $D, \widetilde{D}$ are $p \times p, A$ is $k \times k, \widetilde{A}$ is $(\delta+k) \times$ $(\delta+k)$, defines a mapping

$$
G(z) \rightarrow \widetilde{G}(z)=\widetilde{D}+\widetilde{C}\left(z I_{\delta+k}-\widetilde{A}\right)^{-1} \widetilde{B} .
$$


This mapping coincides with the linear fractional transformation $\widetilde{G}=T_{\Phi_{\mathcal{U}, \mathcal{V}}}(G)$, associated with the $2 p \times 2 p J$-lossless function

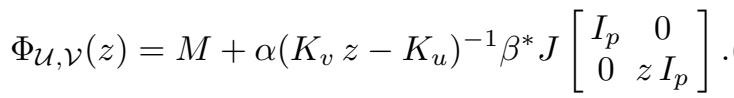

Proof. The case $\delta=1$ has been studied in Hanzon et al. [2004]. It is easily verified that (16) defines a mapping since the function $\widetilde{G}(z)$ does not depend on the choice of the minimal realization $(A, B, C, D)$ of $G(z)$.

We shall use a well-known formula for the inverse of a block matrix [Dym, 1989, sec.0.2]. Assuming that the block $d$ is invertible, the inverse of a block matrix is given by

$$
\begin{aligned}
& {\left[\begin{array}{ll}
a & b \\
c & d
\end{array}\right]^{-1}} \\
& =\left[\begin{array}{cc}
\left(a^{\times}\right)^{-1} & -\left(a^{\times}\right)^{-1} b d^{-1} \\
-d^{-1} c\left(a^{\times}\right)^{-1} & {\left[d^{-1}+d^{-1} c\left(a^{\times}\right)^{-1} b d^{-1}\right]}
\end{array}\right]
\end{aligned}
$$

where $a^{\times}=a-b d^{-1} c$ is known as the Schur complement of $a$. In particular, if

$$
\Gamma(z)=\left[\begin{array}{lc}
\widetilde{D} & \widetilde{C} \\
\widetilde{B} & \left(\widetilde{A}-z I_{\delta+k}\right)
\end{array}\right]
$$

then $\widetilde{G}(z)^{-1}=\left[\begin{array}{ll}I_{p} & 0\end{array}\right] \Gamma(z)^{-1}\left[\begin{array}{c}I_{p} \\ 0\end{array}\right]$. By $(16)$

$$
\begin{gathered}
\Gamma(z)=\left[\begin{array}{cc}
\mathcal{U} & 0 \\
0 & I_{k}
\end{array}\right]\left[\begin{array}{ccc}
D & 0 & C \\
0 & I_{\delta} & 0 \\
B & 0 & A
\end{array}\right]\left[\begin{array}{cc}
\mathcal{V}^{*} & 0 \\
0 & I_{k}
\end{array}\right]-\left[\begin{array}{cc}
0 & 0 \\
0 & z I_{\delta+k}
\end{array}\right] \\
=\Gamma_{0}(z)\left[\begin{array}{cc}
\mathcal{V}^{*} & 0 \\
0 & I_{k}
\end{array}\right], \\
\Gamma_{0}(z)=\left[\begin{array}{ccc}
M_{u} D & \alpha_{u} & M_{u} C \\
\beta_{u}^{*} D-z \beta_{v}^{*} & K_{u}-z K_{v} & \beta_{u}^{*} C \\
B & 0 & A-z I_{k}
\end{array}\right] .
\end{gathered}
$$

The block matrix

$$
d=\left[\begin{array}{cc}
K_{u}-z K_{v} & \beta_{u}^{*} C \\
0 & A-z I_{k}
\end{array}\right]
$$

is invertible and the Schur complement of $M_{u} D$ can be computed as

$$
\begin{aligned}
& M_{u} D-\left[\begin{array}{ll}
\alpha_{u} & M_{u} C
\end{array}\right] d^{-1}\left[\begin{array}{c}
\beta_{u}^{*} D-z \beta_{v}^{*} \\
B
\end{array}\right] \\
& =\Phi_{11}(z) G(z)+\Phi_{12}(z),
\end{aligned}
$$

where $\Phi_{11}(z)=M_{u}-\alpha_{u}\left(K_{u}-K_{v} z\right)^{-1} \beta_{u}^{*}$ and $\Phi_{12}(z)=+\alpha_{u}\left(K_{u}-K_{v} z\right)^{-1} \beta_{v}^{*} z$ are precisely the blocks of the function defined by (17). Still using (18) to compute $\Gamma_{0}(z)^{-1}$, we get

$\widetilde{G}(z)^{-1}=\left[\begin{array}{lll}M_{v} & \alpha_{v} & 0\end{array}\right]\left[\begin{array}{c}\left(\Phi_{11} G+\Phi_{12}\right)^{-1} \\ -d^{-1} c\left(\Phi_{11} G+\Phi_{12}\right)^{-1}\end{array}\right]$, which gives

$$
\widetilde{G}(z)^{-1}=\left(\Phi_{21} G+\Phi_{22}\right)\left(\Phi_{11} G+\Phi_{12}\right)^{-1},
$$

or equivalently $\widetilde{G}=T_{\Phi_{\mathcal{U}, \mathcal{V}}}(G)$. It can be easily established that

$$
\begin{aligned}
& J-\Phi_{\mathcal{U}, \mathcal{V}}(z) J \Phi_{\mathcal{U}, \mathcal{V}}(\lambda)^{*} \\
& =(1-\bar{\lambda} z) \alpha\left(K_{v} z-K_{u}\right)^{-1}\left(K_{v} \lambda-K_{u}\right)^{-*} \alpha^{*},
\end{aligned}
$$

and thus $\Phi_{\mathcal{U}, \mathcal{V}}$ is $J$-lossless.

Remark. A state-space formula of the form (16) associated with some linear fractional transformation has been used in Horiguchi [1999] to describe all the positive real functions which interpolate given input-output characteristics.

Proposition 2. Let $(W, U, V)$ be some admissible Nudelman data set. There exist unitary $(p+\delta) \times$ $(p+\delta)$ matrices $\mathcal{U}$ and $\mathcal{V}$ and a $2 p \times 2 p$ constant $J$-unitary matrix $H_{\mathcal{U}, \mathcal{V}}$ such that

$$
\Theta_{W, U, V} H_{\mathcal{U}, \mathcal{V}}=\Phi_{\mathcal{U}, \mathcal{V}}
$$

Proof. If (19) is satisfied, since $\Theta_{W, U, V}(1)=I_{2 p}$, the matrix $H_{\mathcal{U}, \mathcal{V}}$ must be given by

$$
H_{\mathcal{U}, \mathcal{V}}=M+\alpha\left(K_{v}-K_{u}\right)^{-1} \beta^{*} J .
$$

Moreover, the function $\Phi_{\mathcal{U}, \mathcal{V}}$ cannot have a pole on the circle and can be rewritten

$$
\begin{aligned}
& \Phi_{\mathcal{U}, \mathcal{V}}(z) H_{\mathcal{U}, \mathcal{V}}^{-1}= \\
& {\left[I_{2 p}-(z-1) \alpha\left(K_{v} z-K_{u}\right)^{-1}\left(K_{v}-K_{u}\right)^{-*} \alpha^{*} J\right] .}
\end{aligned}
$$

The representation (10) of a $J$-lossless function being unique up to a similarity transformation (Ball et al. [1990]), there must exist a transformation $T$ such that $P=T^{*} T, K_{u} K_{v}^{-1}=T W T^{-1}$, $\alpha K_{v}^{-1}=C T^{-1}$. The matrix $T$ is thus a square root of $P$. Since the matrix $\mathcal{V}$ must be unitary, we must have $\alpha_{v}^{*} \alpha_{v}+K_{v}^{*} K_{v}=I_{\delta}$, or else

$$
\left(T^{-*} V^{*} V T^{-1}+I_{\delta}\right)^{-1}=K_{v} K_{v}^{*} .
$$

The matrix $T^{-*} V^{*} V T^{-1}+I_{\delta}$ being positive definite, this equation has solutions and $K_{v}$ being one of these, we can set

$$
\left\{\begin{aligned}
\alpha_{u} & =\tilde{U} K_{v} \\
K_{u} & =\tilde{W} K_{v} \\
\alpha_{v} & =\tilde{V} K_{v}
\end{aligned}\right.
$$

in which

$$
\left\{\begin{array}{rl}
\tilde{U} & =U T^{-1} \\
\tilde{W} & =T W T^{-1} \\
\tilde{V} & =V T^{-1}
\end{array} .\right.
$$

These definitions imply that $\alpha_{u}^{*} \alpha_{u}+K_{u}^{*} K_{u}=I_{\delta}$ as required and the columns $\left[\begin{array}{l}\alpha_{u} \\ K_{u}\end{array}\right]$ and $\left[\begin{array}{l}\alpha_{v} \\ K_{v}\end{array}\right]$ can 
be completed into unitary matrices $\mathcal{U}$ and $\mathcal{V}$. The columns $\left[\begin{array}{c}M_{u} \\ \beta_{u}^{*}\end{array}\right]$ and $\left[\begin{array}{c}M_{v} \\ \beta_{v}^{*}\end{array}\right]$ can be determined up to some right $p \times p$ unitary matrices.

\section{EXPLICIT FORMULAS FOR $\mathcal{U}$ AND $\mathcal{V}$.}

An observable pair $(U, W)$ such that $W$ is stable is called output normal if it satisfies

$$
U^{*} U+W^{*} W=I_{\delta} .
$$

Note that two equivalent triples $(W, U, V)$ and $\left(T W T^{-1}, U T^{-1}, V T^{-1}\right)$ give the same interpolation condition (8), so that we can assume that the pair $(U, W)$ in (8) is output normal. From now on, this normalization condition will be imposed to the admissible Nudelman data sets. Explicit formulas for $\mathcal{U}$ and $\mathcal{V}$ can then be given which ensure the smoothness of our parametrization. They have been used for implementation (see section 7.3).

It has been proved that the second block columns of $\mathcal{U}$ and $\mathcal{V}$ are given by (22) and (23) in which $T$ is a square root of $P$ (the solution to (9)) and $K_{v}$ a solution to (21). We choose the uniquely determined Hermitian positive square roots $T=$ $P^{1 / 2}$ and $K_{v}=\left(I_{\delta}+\tilde{V}^{*} \tilde{V}\right)^{-1 / 2}$. It remains to specify the completion of these block columns into unitary matrices. The matrix $M_{v}$ satisfies

$$
M_{v} M_{v}^{*}=I_{p}-\tilde{V}\left(I_{\delta}+\tilde{V}^{*} \tilde{V}\right)^{-1} \tilde{V}^{*}
$$

and it is easily seen that

$$
I_{p}-\tilde{V}\left(I_{\delta}+\tilde{V}^{*} \tilde{V}\right)^{-1} \tilde{V}^{*}=\left(I_{p}+\tilde{V} \tilde{V}^{*}\right)^{-1}
$$

is positive definite. Thus we can choose

$$
M_{v}=\left(I_{p}+\tilde{V} \tilde{V}^{*}\right)^{-1 / 2},
$$

and thus $\beta_{V}^{*}=-\tilde{V}^{*}\left(I_{p}+\tilde{V} \tilde{V}^{*}\right)^{-1 / 2}$, so that we can set

$\mathcal{V}=\left[\begin{array}{cc}\left(I_{p}+\tilde{V} \tilde{V}^{*}\right)^{-1 / 2} & \tilde{V}\left(I_{\delta}+\tilde{V}^{*} \tilde{V}\right)^{-1 / 2} \\ -\tilde{V}^{*}\left(I_{p}+\tilde{V} \tilde{V}^{*}\right)^{-1 / 2} & \left(I_{\delta}+\tilde{V}^{*} \tilde{V}\right)^{-1 / 2}\end{array}\right]$

The construction of a matrix $\mathcal{U}$ is more involved since the matrix $I_{p}-\alpha_{u} \alpha_{u}^{*}$ may fail to be positive definite. This is the case for example when $W$ is the zero matrix, then $\alpha_{u}=U$ and $I_{p}-U U^{*}$ is not invertible. However, when $V$ is zero, since $W$ is stable, $I_{\delta}-W^{*}$ is invertible and there is a simple way to construct a unitary matrix

$$
\begin{gathered}
\mathcal{U}_{0}=\left[\begin{array}{cc}
X & U \\
Y & W
\end{array}\right] \\
\left\{\begin{array}{l}
X=I_{p}-U\left(I_{\delta}-W^{*}\right)^{-1} U^{*} \\
Y=\left(I_{\delta}-W\right)\left(I_{\delta}-W^{*}\right)^{-1} U^{*}
\end{array}\right.
\end{gathered}
$$

Consider the matrix

$$
\left[\begin{array}{cc}
I_{p} & 0 \\
0 & T
\end{array}\right] \mathcal{U}_{0}\left[\begin{array}{cc}
I_{p} & 0 \\
0 & T^{-1}
\end{array}\right]=\left[\begin{array}{cc}
X & \tilde{U} \\
T Y & \tilde{W}
\end{array}\right] .
$$

The problem is now to find a right factor of the form $\left[\begin{array}{cc}\star & 0 \\ \star & K_{v}\end{array}\right]$ which makes it into a unitary matrix. Let

$$
\left[\begin{array}{cc}
N & L^{*} \\
L & K
\end{array}\right]=\left[\begin{array}{cc}
X & \tilde{U} \\
T Y & \tilde{W}
\end{array}\right]^{*}\left[\begin{array}{cc}
X & \tilde{U} \\
T Y & \tilde{W}
\end{array}\right]
$$

A classical method consists of writing a Cholesky factorization using the following well-known factorization of a $(p+\delta) \times(p+\delta)$ block matrix

$$
\begin{aligned}
{\left[\begin{array}{cc}
N & L^{*} \\
L & K
\end{array}\right] } & =\left[\begin{array}{cc}
I_{p} & L^{*} K^{-1} \\
0 & I_{\delta}
\end{array}\right]\left[\begin{array}{cc}
Z^{-1} & 0 \\
0 & K
\end{array}\right]\left[\begin{array}{cc}
I_{p} & 0 \\
K^{-1} L & I_{\delta}
\end{array}\right] \\
& =\left[\begin{array}{cc}
Z^{-1 / 2} & 0 \\
K^{-1 / 2} L & K^{1 / 2}
\end{array}\right]^{*}\left[\begin{array}{cc}
Z^{-1 / 2} & 0 \\
K^{-1 / 2} L & K^{1 / 2}
\end{array}\right]
\end{aligned}
$$

where $Z=\left(N-L^{*} K^{-1} L\right)^{-1}$. By (18), $Z$ is the left upper block of

$$
\left[\begin{array}{cc}
N & L^{*} \\
L & K
\end{array}\right]^{-1}=\left[\begin{array}{cc}
I_{p} & 0 \\
0 & T
\end{array}\right] \mathcal{U}_{0}^{*}\left[\begin{array}{cc}
I_{p} & 0 \\
0 & P^{-1}
\end{array}\right] \mathcal{U}_{0}\left[\begin{array}{cc}
I_{p} & 0 \\
0 & T^{*}
\end{array}\right]
$$

and can be computed as

$$
Z=X^{*} X+Y^{*} P^{-1} Y .
$$

The matrices $L$ and $K$ are given by

$$
\begin{aligned}
L & =\tilde{U}^{*} X+\tilde{W}^{*} T Y \\
K & =\tilde{U}^{*} \tilde{U}+\tilde{W}^{*} \tilde{W}=I_{\delta}+\tilde{V}^{*} \tilde{V} .
\end{aligned}
$$

Note that the matrices $Z$ and $K$ are actually positive definite and that $K^{-1 / 2}=K_{v}$ as desired. Thus, we can set

$$
\mathcal{U}=\left[\begin{array}{cc}
X & \tilde{U} \\
T Y & \tilde{W}
\end{array}\right]\left[\begin{array}{cc}
Z^{1 / 2} & 0 \\
-K^{-1} L Z^{1 / 2} & K^{-1 / 2}
\end{array}\right]
$$

This proves the following result:

Proposition 3. Let $(W, U, V)$ be some admissible Nudelman data set satisfying (24). Define the map $\tau:(W, U, V) \rightarrow(\mathcal{U}, \mathcal{V})$, where $\mathcal{U}$ and $\mathcal{V}$ are the unitary matrices given by (31) and (25), where $\widetilde{U}$, $\widetilde{V}, \widetilde{W}$ are given by (23), K, $L, Z$ by $(30),(29),(28)$ and $X, Y$ by (27), in all of which $T$ is the positive square root of $P$, the solution to (9).

Then, the $J$-lossless function

$$
\hat{\Theta}_{W, U, V}=\Theta_{W, U, V} H_{\mathcal{U}, \mathcal{V}},
$$

$H_{\mathcal{U}, \mathcal{V}}$ being given by $(20)$, coincides with $\Phi_{\mathcal{U}, \mathcal{V}}$.

Let $\Lambda$, $\Pi$ be $p \times p$ unitary matrices and $\Sigma$ be a $\delta \times \delta$ unitary matrix. Noting that $\left(\Lambda Z \Lambda^{*}\right)^{1 / 2}=$ $\Lambda Z^{1 / 2} \Lambda^{*}$, it is easily verified that 


$$
\begin{aligned}
& \tau(W, \Lambda U, \Pi V) \\
& =\left(\left[\begin{array}{cc}
\Lambda & 0 \\
0 & I_{\delta}
\end{array}\right] \mathcal{U}\left[\begin{array}{cc}
\Lambda^{*} & 0 \\
0 & I_{\delta}
\end{array}\right],\left[\begin{array}{cc}
\Pi & 0 \\
0 & I_{\delta}
\end{array}\right] \mathcal{V}\left[\begin{array}{cc}
\Pi^{*} & 0 \\
0 & I_{\delta}
\end{array}\right]\right),
\end{aligned}
$$

so that the $J$-lossless function $\hat{\Theta}_{W, U, V}$ also satisfies (12) and (13).

We also have that

$$
\begin{aligned}
& \tau\left(\Sigma^{*} W \Sigma, U \Sigma, V \Sigma\right) \\
& =\left(\left[\begin{array}{cc}
I_{p} & 0 \\
0 & \Sigma^{*}
\end{array}\right] \mathcal{U}\left[\begin{array}{cc}
I_{p} & 0 \\
0 & \Sigma
\end{array}\right],\left[\begin{array}{cc}
I_{p} & 0 \\
0 & \Sigma^{*}
\end{array}\right] \mathcal{V}\left[\begin{array}{cc}
I_{p} & 0 \\
0 & \Sigma
\end{array}\right]\right),
\end{aligned}
$$

so that

$$
\hat{\Theta}_{\Sigma^{*} W \Sigma, U \Sigma, V \Sigma}=\hat{\Theta}_{W, U, V}
$$

Corollary 1. A unitary matrix realization $\widetilde{R}$ of $\widetilde{G}=T_{\hat{\Theta}_{W, U, V}}(G)$ can be computed from a unitary matrix realization $R$ of $G(z)$ by (16) in which $\mathcal{U}$ and $\mathcal{V}$ are the unitary matrices $(\mathcal{U}, \mathcal{V})=$ $\tau(W, U, V)$ defined in Proposition 3.

Remark. Instead of $\mathcal{U}$ and $\mathcal{V}$, we may have chosen

$$
\begin{aligned}
& \hat{\mathcal{U}}=\left[\begin{array}{cc}
I_{p} & 0 \\
0 & O_{1}
\end{array}\right] \mathcal{U}\left[\begin{array}{cc}
H_{1} & 0 \\
0 & O_{2}
\end{array}\right] \\
& \hat{\mathcal{V}}=\left[\begin{array}{cc}
I_{p} & 0 \\
0 & O_{1}
\end{array}\right] \mathcal{V}\left[\begin{array}{cc}
H_{2} & 0 \\
0 & O_{2}
\end{array}\right],
\end{aligned}
$$

in which $\mathrm{O}_{1}, \mathrm{O}_{2}, \mathrm{H}_{1}$ and $\mathrm{H}_{2}$ are unitary matrices: (1) the matrix $O_{1}$ corresponds to the choice of $O_{1} P^{1 / 2}$ instead of $P^{1 / 2}$. This choice leave the linear fractional transformation unchanged $\left(\Phi_{\hat{\mathcal{U}}, \hat{\mathcal{V}}}=\Phi_{\mathcal{U}, \mathcal{V}}\right)$, but changes the realization $\widetilde{R}=$ $(\widetilde{A}, \widetilde{B}, \widetilde{C}, \widetilde{D})$ into $\left(O_{1} \widetilde{A} O_{1}^{*}, O_{1} \widetilde{B}, \widetilde{C} O_{1}^{*}, \widetilde{D}\right)$, a similar one.

(2) the choice of $K_{v}=\left(\tilde{V}^{*} \tilde{V}+I_{p}\right)^{-1 / 2} O_{2}$ instead of $\left(\tilde{V}^{*} \tilde{V}+I_{p}\right)^{-1 / 2}$ has no effect.

(3) The unitary matrices $H_{1}$ and $H_{2}$ correspond to another completion of the first columns of $\mathcal{V}$ and $\mathcal{U}$. This choice changes the linear fractional transformation since

$$
\Phi_{\hat{\mathcal{U}}, \hat{\mathcal{V}}}=\Phi_{\mathcal{U}, \mathcal{V}}\left[\begin{array}{cc}
H_{1} & 0 \\
0 & H_{2}
\end{array}\right]
$$

and thus produces a non-similar realization.

Remark. Note that if $V=0$, since the pair $(U, W)$ satisfies $U^{*} U+W^{*} W=I_{\delta}$, we have that $P=I_{\delta}$ and $T=I_{\delta}$ too. Thus, $\mathcal{U}=\mathcal{U}_{0}$ defined by (26) and $\mathcal{V}=I_{p+\delta}$, so that the recursion (16) becomes

$$
\left[\begin{array}{c|c}
\widetilde{D} & \widetilde{C} \\
\hline \widetilde{B} & \widetilde{A}
\end{array}\right]=\left[\begin{array}{c|cc}
M_{u} D & U & M_{u} C \\
\hline \beta_{u}^{*} D & W & \beta_{u}^{*} C \\
B & 0 & A
\end{array}\right]
$$

\section{CHARTS FROM A SCHUR ALGORITHM.}

Recall that a manifold is a topological space that looks locally like the "ordinary" Euclidean space $\mathbb{R}^{N}$ : near every point of the space, we have a coordinate system or chart. The number $N$ is the dimension of the manifold. It has been proved in [Alpay et al., 1994, Th.2.2] that $\mathcal{L}_{n}^{p}$ is a smooth manifold of dimension $p^{2}+2 n p$ embedded in the Hardy space $H_{q}^{p \times p}$, for $1 \leq q \leq \infty$ $\left(\mathcal{R} \mathcal{L}_{n}^{p}\right.$ is a smooth manifold of dimension $\frac{p(p-1)}{2}+$ $n p)$. The topology on $\mathcal{L}_{n}^{p}$ is that induced by the $L^{q}$ norm on $H_{q}^{p \times p}$. In Alpay et al. [1994] atlases of charts have been constructed from a Schur algorithm associated with Nevanlinna-Pick interpolation. We generalize this construction to the case of Nudelman interpolation.

Let $\sigma=\left(\left(U_{1}, W_{1}\right),\left(U_{2}, W_{2}\right), \ldots,\left(U_{l}, W_{l}\right)\right)$ be a sequence of output normal pairs (see section 5 ), $W_{j}$ is $n_{j} \times n_{j}, U_{j}$ is $p \times n_{j}$, and

$$
\sum_{j=1}^{l} n_{j}=n
$$

From a given lossless function $G(z)$ of degree $n$, a sequence of lossless functions of decreasing degree $G_{l}(z)=G(z), G_{l-1}(z), \ldots$ can be constructed following a Schur algorithm: assume that $G_{j}(z)$ has been constructed and put

$$
V_{j}=\frac{1}{2 i \pi} \int_{\mathbf{T}} G_{j}^{\sharp}(z) U_{j}\left(z I_{n_{j}}-W_{j}\right)^{-1} d z .
$$

If the solution $P_{j}$ to the symmetric Stein equation

$$
P_{j}-W_{j}^{*} P_{j} W_{j}=U_{j}^{*} U_{j}-V_{j}^{*} V_{j}
$$

is positive definite, then from Theorem 2, a lossless function $G_{j-1}(z)$ is defined by

$$
G_{j}=T_{\hat{\Theta}_{W_{j}, U_{j}, V_{j}}}\left(G_{j-1}\right)
$$

If $P_{j}$ is not positive definite, the construction stops.

A chart $(\mathcal{D}, \phi)$ of $\mathcal{L}_{n}^{p}$ is attached with a sequence $\sigma$ of output normal pairs and with a chart $(\mathcal{W}, \psi)$ of $\mathbb{U}(p)$ as follows:

A function $G(z) \in \mathcal{L}_{n}^{p}$ belongs to the domain $\mathcal{D}$ of the chart if the Schur algorithm allows to construct a complete sequence of lossless functions,

$$
G(z)=G_{l}(z), G_{l-1}(z) \ldots, G_{0},
$$

where $G_{0}$ is a constant lossless matrix in $\mathcal{W} \subset$ $\mathbb{U}(p)$.

The local coordinate map $\phi$ is defined by

$$
\phi: G(z) \in \mathcal{D} \rightarrow\left(V_{1}, V_{2}, \ldots, V_{l}, \psi\left(G_{0}\right)\right),
$$


and the interpolation matrices $V_{j}$ are called the Schur parameters of the function in the chart.

Theorem 3. A family of charts $(\mathcal{D}, \phi)$ defines an atlas of $\mathcal{L}_{n}^{p}$ provided the union of their domains covers $\mathcal{L}_{n}^{p}$.

Proof. The proof is analogous to the proof of Th.3.5 in Alpay et al. [1994]. The domain $\mathcal{D}$ of a chart is open and the map $\phi$ is a diffeomorphism. This relies on the fact that $\hat{\Theta}_{W, U, V}$ depends smoothly on the entries of $V$.

Atlases for the quotient $\mathcal{L}_{n}^{p} / \mathbb{U}(p)$ are obtained using the properties (12) and (13). If $G(z)$ has Schur parameters $\left(V_{1}, V_{2}, \ldots, V_{l}\right)$ and constant unitary matrix $G_{0}$ in a given chart, and if $\Pi \in \mathbb{U}(p)$, then $G(z) \Pi^{*}$ has Schur parameters $\left(\Pi V_{1}, \Pi V_{2}, \ldots, \Pi V_{l}\right)$ and constant unitary matrix $G_{0} \Pi^{*}$ in the same chart. The quotient can be performed within a chart by imposing the last constant lossless matrix $G_{0}$ in the Schur algorithm to be the identity matrix.

In a chart, a balanced realization $R$ of $G(z)=$ $\phi^{-1}\left(V_{1}, V_{2}, \ldots, \psi\left(G_{0}\right)\right)$ can be computed from the parameters using the Schur sequence: let $R_{0}=$ $G_{0}$, a realization $R_{j}$ of $G_{j}(z)$ is obtained from a realization $R_{j-1}$ of $G_{j-1}(z)$ by formula (16) in which $(\mathcal{U}, \mathcal{V})=\left(\mathcal{U}_{j}, \mathcal{V}_{j}\right)=\tau\left(W_{j}, U_{j}, V_{j}\right)$ (see Proposition 3$)$. This process allows to select for each $G(z)$ in the domain of the chart a unique balanced realization within the equivalence class, and then the map

$$
R \rightarrow\left(V_{1}, V_{2}, \ldots, \psi\left(G_{0}\right)\right)
$$

is a canonical form. However, the domain of this canonical form is not easily characterized and it is in general difficult to decide if a given realization is in canonical form with respect to a chart. This can be done in some particular situations that will be studied in the following section.

\section{SOME PARTICULAR ATLASES.}

We describe three atlases which all present some interest from the optimization viewpoint. The first one is for complex functions and it involves only Schur steps in which the degree is increased by one. It allows for a search strategy of local minima by induction on the degree, which can be very helpful in some difficult optimization problems. The second one is the analog for realvalued functions. The third one involves only one Schur step and provides very simple and natural canonical forms.

A chart in which all the Schur parameters $V_{j}$ are zero matrices for $G(z)$ is called an adapted chart for $G(z)$. Such a chart presents a great interest from an optimization viewpoint. The optimization process starts, in an adapted chart, at the origin and thus far from the boundary where a change of chart is necessary. For each atlas, a simple method to find an adapted chart is given.

\subsection{An atlas for $\mathcal{L}_{n}^{p}$ (complex lossless functions)}

Consider the charts associated with sequences of output normal pairs $\left(u_{1}, w_{1}\right),\left(u_{2}, w_{2}\right), \ldots,\left(u_{n}, w_{n}\right)$ in which the $w_{j}$ 's are complex numbers. In this case, the Nudelman interpolation condition (8) can be rewritten as a Nevanlinna-Pick interpolation condition

$$
G\left(1 / \bar{w}_{j}\right)^{*} u_{j}=v_{j}
$$

This is the atlas described in Hanzon et al. [2004]. However, the normalization conditions differ. In Hanzon et al. [2004] the $p$-vectors $u_{j}$ have norm one, while in this work, the pairs $\left(u_{j}, w_{j}\right)$ are output normal (24).

Remark. Note that, when $n_{j}>1$ the normalization condition $U_{j}$ unitary (a possible generalization of $\left\|u_{j}\right\|=1$ ) cannot be chosen since the matrix $U_{j}^{*} U_{j}$ can be singular.

In view of (34), an adapted chart can be computed from a realization in Schur form.

Lemma 1. Let $\widetilde{G}(z) \in \mathcal{L}_{n}^{p}$ and let $\widetilde{R}=(\widetilde{A}, \widetilde{B}, \widetilde{C}, \widetilde{D})$ be a balanced realization of $G(z)$ in Schur form $(\widetilde{A}$ upper triangular). Let

$$
\widetilde{A}=\left[\begin{array}{cc}
w & a^{*} \\
0 & A
\end{array}\right], \quad \widetilde{B}=\left[\begin{array}{l}
b^{*} \\
B
\end{array}\right], \quad \widetilde{C}=[u \hat{C}],
$$

where $w \in \mathbb{C}, u, b \in \mathbb{C}^{p}$, and $a \in \mathbb{C}^{n-1}$. Then, $\widetilde{G}=T_{\hat{\Theta}_{w, u, 0}}(G)$ for some lossless function $G(z)$. A realization $R$ of $G(z)$ can be computed by reverting (16). It is still in Schur form and given by $R=(A, B, C, D)$, where

$$
\begin{aligned}
& C=\hat{C}+(1-w)^{-1} u a^{*} \\
& D=\widetilde{D}+(1-w)^{-1} u b^{*}
\end{aligned}
$$

This process can be repeated. It provides a sequence of output normal pairs $\left(u_{j}, w_{j}\right)$, the $w_{j}$ 's being the eigenvalues of $\widetilde{A}$. In the corresponding chart the Schur parameters of $G(z)$ are the zero $p$-vectors $v_{n}=\ldots=v_{1}=0$.

\subsection{An atlas for $\mathcal{R} \mathcal{L}_{n}^{p}$ (real lossless functions)}

To deal with real functions we consider the charts associated with sequences of output normal pairs 
$\left(U_{1}, W_{1}\right),\left(U_{2}, W_{2}\right), \ldots,\left(U_{n}, W_{n}\right)$ in which the $W_{j}$ 's are either real numbers or real $2 \times 2$ matrices with complex conjugate eigenvalues and the $U_{j}$ 's are real matrices. The parameters (the matrices $\left.V_{j}\right)$ are then restricted to be real.

As previously, an adapted chart for a given lossless function $\widetilde{G}(z) \in \mathcal{R}_{\mathcal{L}_{n}^{p}}$ can be obtained from a realization in real Schur form.

Lemma 2. Let $\widetilde{G}(z) \in \mathcal{R L}_{n}^{p}$ and let $\widetilde{R}=$ $(\widetilde{A}, \widetilde{B}, \widetilde{C}, \widetilde{D})$ be a balanced realization of $G(z)$ in real Schur form

$$
\widetilde{A}=\left[\begin{array}{cccc}
W_{l} & \star & \cdots & \star \\
0 & W_{l-1} & \ddots & \vdots \\
\vdots & \ddots & \ddots & \star \\
0 & \cdots & 0 & W_{1}
\end{array}\right]
$$

where for $j=1, \ldots, l, W_{j}$ is either a real number or a $2 \times 2$ block with complex conjugate eigenvalues. Let

$$
\widetilde{A}=\left[\begin{array}{cc}
W_{l} & \hat{A}^{*} \\
0 & A
\end{array}\right], \quad \widetilde{C}=\left[\begin{array}{ll}
U_{l} & \hat{C}
\end{array}\right], \quad \widetilde{B}=\left[\begin{array}{l}
\hat{B}^{*} \\
B
\end{array}\right],
$$

where $U_{l}$ and $\hat{B}$ are $p \times n_{l}, n_{l}$ being the size of $W_{l}$. Then, $\widetilde{G}=T_{\hat{\Theta}_{W_{l}, U_{l}, 0}}(G)$ for some lossless function $G(z)$. A realization $R$ of $G(z)$ can be computed by reverting (16). It is in Schur form and given by $R=(A, B, C, D)$, where

$$
\begin{aligned}
& C=\hat{C}+\left(I_{n_{l}}-W_{l}\right)^{-1} U_{l} \hat{A}^{*} \\
& D=\widetilde{D}+\left(I_{n_{l}}-W_{l}\right)^{-1} U_{l} \hat{B}^{*}
\end{aligned}
$$

Repeating this process, we get a sequence of output normal pairs $\left(U_{j}, W_{j}\right)$, the $W_{j}$ 's being the diagonal blocks of $\widetilde{A}$, that index a chart in which the Schur parameters (which are now matrices of different sizes) of $G(z)$ are all zero matrices.

Remark. The Schur algorithm attached with $G(z)$ in such an adapted chart yields a Potapov factorization for real lossless functions

$$
G(z)=B_{l}(z) B_{l-1}(z) \cdots B_{1}(z)
$$

where $B_{j}$ is the real-valued lossless function

$$
\begin{aligned}
& B_{j}(z)= \\
& I_{p}-(z-1) U_{j}\left(z I_{n_{j}}-W_{j}\right)^{-1}\left(I_{n_{j}}-W_{j}^{*}\right)^{-1} U_{j}^{*} .
\end{aligned}
$$

\subsection{Lossless mutual encoding.}

For this atlas, we consider the charts associated with a single output normal pair $(U, W)$ in which $W$ is $n \times n$ and $U$ is $p \times n$. In this case, a solution to (8) can be directly characterized in state space form and formula (16) recovered independently from the Schur algorithm.

Proposition 4. Let $G(z)=D+C\left(z I_{n}-A\right)^{-1} B$ be a balanced realization of $G(z) \in \mathcal{L}_{n}^{p}$. Let $Q$ be the unique solution to the Stein equation

$$
Q-A^{*} Q W=C^{*} U
$$

Then, the interpolation value $V$ in (8) and the solution $P$ to $(9)$ are given by

$$
\begin{gathered}
V=D^{*} U+B^{*} Q W, \\
P=Q^{*} Q .
\end{gathered}
$$

The unitary realization matrix $R=\left[\begin{array}{ll}D & C \\ B & A\end{array}\right]$ of $G(z)$ can be computed as

$$
R=\hat{\mathcal{U}}\left[\begin{array}{cc}
G_{0} & 0 \\
0 & I_{n}
\end{array}\right] \hat{\mathcal{V}}^{*}, \quad G_{0} \in \mathbb{U}(p),
$$

in which $\hat{\mathcal{U}}$ and $\hat{\mathcal{V}}$ are unitary matrices given as in Proposition 3 by $(31)$ and $(25)$, where $\widetilde{U}, \widetilde{V}, \widetilde{W}$ are given by (23), K, L, $Z$ by $(30),(29),(28)$ and $X, Y$ by (27), but in which the square root $T$ of $P$ is now chosen to be $Q$.

Proof. Since $G^{\sharp}(z)=D^{*}+B^{*}\left(\frac{1}{z} I_{n}-A^{*}\right)^{-1} C^{*}$, the contour integral (8) can be computed as

$$
\begin{aligned}
V= & \frac{1}{2 i \pi} \int_{\mathbf{T}} G^{\sharp}(z) U\left(z I_{n}-W\right)^{-1} d z \\
V= & \frac{1}{2 i \pi} \int_{\mathbf{T}} D^{*} U\left(\sum_{j=0}^{\infty}\left(z^{-1} W\right)^{j}\right) \\
& +B^{*} z\left(\sum_{j=0}^{\infty}\left(z A^{*}\right)^{j}\right) C^{*} U\left(\sum_{j=0}^{\infty} z^{-j} W^{j}\right) \frac{d z}{z}, \\
= & D^{*} U+B^{*}\left(\sum_{j=0}^{\infty}\left(A^{*}\right)^{j} C^{*} U W^{j}\right) W .
\end{aligned}
$$

Since $Q$ is given by the convergent series $Q=$ $\sum_{j=0}^{\infty}\left(A^{*}\right)^{j} C^{*} U W^{j},(36)$ is satisfied.

Consider a unitary completion of the column $\left[\begin{array}{c}U \\ W\end{array}\right]$, for example the matrix $\mathcal{U}_{0}$ given by (26). Here, $\mathcal{U}_{0}$ and the unitary realization matrix $R$ have the same size and formulas (35) and (36) can be rewritten in a matrix form

$$
R^{*}\left[\begin{array}{cc}
I_{p} & 0 \\
0 & Q
\end{array}\right] \mathcal{U}_{0}=\left[\begin{array}{c}
\star V \\
\star Q
\end{array}\right]=\Upsilon
$$

The matrix $R$ being unitary, we have that

$$
\Upsilon^{*} \Upsilon=\mathcal{U}_{0}^{*}\left[\begin{array}{cc}
I_{p} & 0 \\
0 & Q^{*} Q
\end{array}\right] \mathcal{U}_{0}=\left[\begin{array}{ll}
\star & \star \\
\star & V^{*} V+Q^{*} Q
\end{array}\right]
$$


so that $U^{*} U+W^{*} Q^{*} Q W=V^{*} V+Q^{*} Q$, which proves (37).

We shall use the computations of section 4 , with $\delta=n$ and $T=Q$ instead of the Hermitian positive square root $P^{1 / 2}$. From (38) we get

$$
R^{*}\left[\begin{array}{cc}
I_{p} & 0 \\
0 & Q
\end{array}\right] \mathcal{U}_{0}\left[\begin{array}{cc}
I_{p} & 0 \\
0 & Q^{-1}
\end{array}\right]=\left[\begin{array}{cc}
\star & \widetilde{V} \\
\star & I_{p}
\end{array}\right]
$$

and if $\hat{\mathcal{U}}$ is the unitary matrix given by (31),

$$
R^{*} \hat{\mathcal{U}}=\left[\begin{array}{cc}
\star \widetilde{V} K^{-1 / 2} \\
\star & K^{-1 / 2}
\end{array}\right] .
$$

This matrix is unitary and its second block column coincides with that of $\hat{\mathcal{V}}$ given by (25). Thus it must be $\hat{\mathcal{V}}$ up to a right unitary factor of the form $\left[\begin{array}{cc}G_{0}^{*} & 0 \\ 0 & I_{p}\end{array}\right]$, for some unitary matrix $G_{0}$.

Remark. Note that $\Phi_{\hat{\mathcal{U}}, \hat{\mathcal{V}}}=\Phi_{\mathcal{U}, \mathcal{V}}(\mathcal{U}, \mathcal{V}$ defined in Proposition 3 ) and thus $G_{0}$ is the constant unitary matrix in the Schur algorithm $G=T_{\hat{\Theta}_{W, U, V}}\left(G_{0}\right)$.

The domain of a chart and of the associated canonical form can then be easily characterized.

Proposition 5. A lossless function $G(z)$, given by a balanced realization $(A, B, C, D)$ can be parametrized in the chart defined by the pair $(U, W)$ if and only if the solution $Q$ to the Stein equation (35) is positive definite. A realization $\tilde{R}$ is in canonical form with respect to this chart if and only if the solution $Q$ to (35) is $P^{1 / 2}, P$ being a solution of (9).

The invertibility of the matrix $Q$ is a good measure of the quality of the chart, the best choice being $Q=I_{n}$. This choice provides an adapted chart.

Proposition 6. The chart associated with the output normal pair $(C, A)$ in a balanced realization $(A, B, C, D)$ of $G(z)$ is an adapted chart for $G(z)$.

Proof. In this case, $Q=I_{n}$, so that $P=I_{n}$ and $V=0$.

Remark. Let $(\mathcal{D}, \phi), \phi: G(z) \rightarrow\left(V, G_{0}\right)$ be the chart associated with the output normal pair $(U, W)$. Then the chart associated with the pair $\left(U \Sigma, \Sigma^{*} W \Sigma\right)$ has same domain $\mathcal{D}$ and by (33) coordinate map $\phi^{\prime}: G(z) \rightarrow\left(V \Sigma, G_{0}\right)$. In an atlas, these two charts play the same role.

Remark. Equivalence classes of output normal pairs are in bijection with lossless functions in $\mathcal{L}_{n}^{p} / \mathbb{U}(p)$ [Alpay et al., 1994, Cor.2.1]. The unitary completion $\mathcal{U}_{0}$ of the matrix $\left[\begin{array}{c}U \\ W\end{array}\right]$ in (26) defines a lossless function

$$
\Omega(z)=X+U\left(z I_{n}-W\right)^{-1} Y \in \mathcal{L}_{n}^{p} .
$$

The canonical form associated with the pair $(U, W)$ depends on this completion and is in fact attached with an element of $\mathcal{L}_{n}^{p} / \mathbb{U}(p)$. This explains why this section was called lossless mutual encoding.

\section{APPLICATION TO SYSTEM IDENTIFICATION AND CONTROL.}

The first application that we consider is the identification of hyperfrequency filters, made of coupled resonant cavities, that are used in telecommunication satellites for channel multiplexing. The problem is to recover the transfer function of the filter from frequency data. These data are estimate values of the transfer function at pure imaginary points obtained from the steady-state outputs of the filter to harmonic inputs. A first stage, far from being trivial, consists in computing a stable matrix transfer function of high order which agrees with the data. It is achieved by the software Hyperion, also developed at INRIA (Baratchart et al. [1998]). Then a rational $L^{2}$ approximation stage is performed by the software RARL ${ }^{1}$, in which the atlas of section 7.1 is used. Transfer functions of these filters are complex functions since a particular transformation has been used to simplify the model. In Figure 1, a 8th order model of a MIMO $2 \times 2$ hyperfrequency filter is shown, obtained from 800 pointwise data. A longstanding
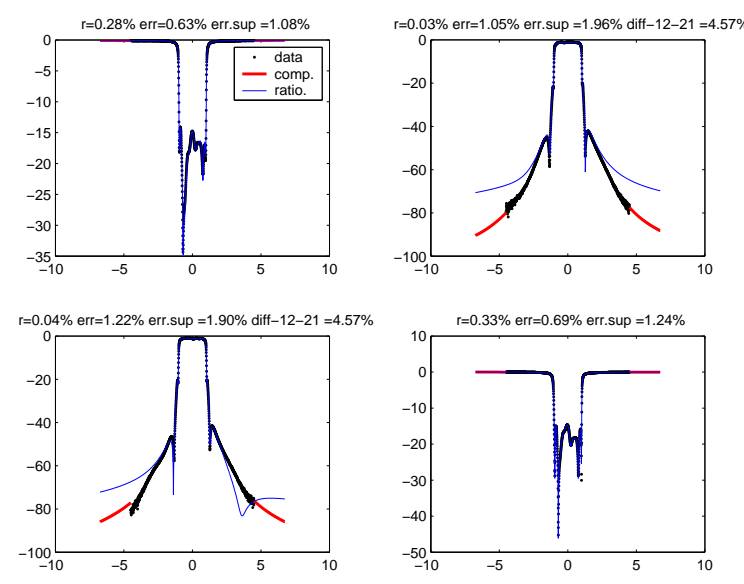

Fig. 1 . CNES $2 \times 2$ hyperfrequency filter: data and approximant at order 8 (Bode diagram).

cooperation with the space agency CNES resulted in a dedicated software PRESTO-HF that wraps both HYPERION and RARL2 into a package which is now fully integrated in the design and tuning process.

1 The software RARL2 is described in Marmorat et al. [2002] and available at the web page http: www-sop.inria.fr/apics/RARL2/rarl2-eng.html . 
This application and more generally filter design, raises interesting new parametrization issues. The physical laws of energy conservation and reciprocity introduce subclasses of transfer functions which play an important role in this domain. These include $J$-inner, Schur (or contractive), positive real and symmetric functions. In another connection, systems having a particular state space form must be handled to account for some physical properties, like for example the coupling geometry of a filter. We think that Schur analysis could help us to describe such subclasses and to pave the bridge between the frequency domain (where specifications are made) and the state-space domain (where the design parameters live). As a first step in this way, a Schur algorithm for symmetric lossless functions, based on a twosided Nudelman interpolation condition, has been presented in Olivi et al. [2005].

The atlas of section 7.3 has been recently implemented in RARL2, the rational $L^{2}$ approximation software. Its effectiveness has been demonstrated on random systems and classical examples from the literature. A promising field of application, in which functions are real-valued, is multi-objective control. In Scherer [2000], revisited in a chainscattering perspective in Drai et al. [2005], it is shown that if the pair $\left(C_{Q}, A_{Q}\right)$ of the Youla parameter $Q(z)=D_{Q}+C_{Q}\left(z I-A_{Q}\right)^{-1} B_{Q}$ is fixed, then the search over the parameters $\left(B_{Q}, D_{Q}\right)$ can be reduced to an efficiently solvable LMI problem. Limiting the search of the parameter $Q(z)$ to the FIR form

$$
Q(z)=Q_{0}+Q_{1} \frac{1}{z}+\ldots+Q_{p} \frac{1}{z^{p}}
$$

provides solutions to the multi-objective control problem. However, this is also the main limitation of the approach as high order expansions might be necessary, due notably to the fact that the poles structure is fixed through the pair $\left(C_{Q}, A_{Q}\right)$. Such a drawback could be avoided if the search was performed over all the parameters $Q(z)$ of fixed McMillan degree. This could be done using the atlas of section 7.3 to parametrize the corresponding pairs $\left(C_{Q}, A_{Q}\right)$. This work is currently under investigation and will be reported later.

\section{REFERENCES}

D. Alpay, L. Baratchart, and A. Gombani. On the differential structure of matrix-valued rational inner functions. Operator Theory : Advances and Applications, 73:30-66, 1994.

J.A. Ball, I. Gohberg, and L. Rodman. Interpolation of rational matrix functions, volume 45 of Operator Theory: Advances and Applications. Birkhäuser, 1990.
L. Baratchart, J. Grimm, J. Leblond, M. Olivi, F. Seyfert, and F. Wielonsky. Identification d'un filtre hyperfréquence par approximation dans le domaine complexe. Technical Report 219, INRIA, 1998.

J. Bruls, C.T. Chou, B.R.J. Haverkamp, and M. Verhaegen. Linear and non-linear system identification using separable least-squares. European Journal of Control, 5(1):p. 116-128, 1999.

R. Drai, J.P. Marmorat, and M. Olivi. A chainscattering approach to LMI multiobjective control. In 16th IFAC World Congress, Prague, July 2005.

H. Dym. J-contractive matrix functions, reproducing kernel spaces and interpolation, volume 71 of $C B M S$ lecture notes. American Mathematical Society, Rhode Island, 1989.

P. Fulcheri and M. Olivi. Matrix rational $\mathrm{H}^{2}$ approximation: a gradient algorithm based on Schur analysis. SIAM Journal on Control and Optimisation, 36, No. 6:2103-2127, 1998.

Y. Genin, P. Van Dooren, T. Kailath, J.-M. Delosme, and M. Morf. On $\Sigma$-lossless transfer functions and related questions. Linear Algebra and its Applications, 50:251-275, 1983.

B. Hanzon and R.L.M. Peeters. Balanced parametrizations of stable SISO all-pass systems in discrete-time. MCSS, 13:240-276, 2000.

B. Hanzon, M. Olivi, and R.L.M. Peeters. Balanced realizations of discrete-time stable allpass systems and the tangential schur algorithm. Technical Report 5111, INRIA, 2004. to appear in Linear Algebra and Appl.

K. Horiguchi. Discrete-time positive real matrix functions interpolating input-output characteristics. IEICE Trans. Fundamentals, E82-A(8): 1608-1618, 1999.

J.-P. Marmorat, M. Olivi, B. Hanzon, and R.L.M. Peeters. Matrix rational $H^{2}$ approximation: a state-space approach using Schur parameters. In Proceedings of the CDC02, Las-Vegas, USA., 2002.

J.-P. Marmorat, M. Olivi, B. Hanzon, and R.L.M. Peeters. Schur parametrizations and balanced realizations of real discrete-time stable allpass systems. In Proceedings of the CDC03, Maui, HAWAI., 2003.

M. Olivi, B. Hanzon, and R. Peeters. A Schur algorithm for symmetric inner functions. In CDC 2005, Seville, Spain, Dec 2005.

R. Peeters, B. Hanzon, and M. Olivi. Canonical lossless state-space systems: staircase forms and the Schur algorithm. In SSSC 2004, Oaxaca, Mexico, Dec 2004.

C. Scherer. An efficient solution to multi-objective control problems with LMI objectives. System and Control Letters, pages 75-84, 2000. 
This figure "BodeRes.png" is available in "png" format from: http://arxiv.org/ps/1012.3335v1 